\title{
Optimal Dispatch of Regional Integrated Heating and Power System Based on Differential Thermal Inertia Model
}

\author{
Shiyuan $\mathrm{Ni}^{1}$, Yi Lin ${ }^{1}$, Defei $\mathrm{Yao}^{2}$, Guilian $\mathrm{Wu}^{1}$, Zehao Wang ${ }^{2}$ and Yan Huang ${ }^{2 *}$ \\ ${ }^{1}$ Power Economic Research Institute of State Grid Fujian Electric Power Co., Ltd., Fuzhou, Fujian, 350000, China \\ ${ }^{2}$ Sichuan Energy Internet Research Institute Tsinghua University, Chengdu, Sichuan, 610000, China
}

\begin{abstract}
As the physical carrier of Energy Internet, regional integrated energy system (RIES) has become an important role for improving comprehensive energy utilization efficiency. First, a subtle thermodynamic model of buildings and water-heating network was constructed based on differential thermal inertia model. Different from the traditional single-layer wall thermal inertial model, this paper constructed multi-layer wall thermal model. Then, an optimization scheme combining electricity and heat was established. The optimization results show that, compared with the traditional single-layer wall thermal inertia model, the proposed multi-layer wall thermal inertia model has better performance. The proposed comprehensive energy optimization scheme can reduce the cost of electricity while maintaining indoor comfort, and can provide a reference for the system operation status for distribution network dispatching.
\end{abstract}

\section{Introduction}

The regional integrated energy system (RIES) is considered to be the main form of energy for future human society. The RIES is mainly composed of energy supply networks, energy exchange links, energy storage links and a large number of users. As an efficient measure to improve energy efficiency, the RIES have gained rapid development in recent years[1].

Over the years buildings have gradually evolved into multi-energy carriers owing to their significant thermal load. In order to achieve higher utilization efficiencies, combined cooling, heating and power (CCHP) systems have been identified as potential solutions[2]. In reference[3], an integrated electricity-gas-heat network model was developed, but the piecewise friction coefficient model of a water-heating network was not considered. Reference[4] uses embedded wall-type pipes to heating the building and improves the building energy storage. Air conditioners are also involved in voltage regulation, demand response. If the air-conditioning load is controllable, an optimized scheduling model can be proposed, which can not only reduce the peak load, but also dispatch quickly and accurately in an emergency[5]. At the same time, due to the thermal inertia of the building, the change of air conditioning load in a short time will not affect people comfort[6]. Therefore, when the thermal inertia process is modeled more accurately, the airconditioning load can be effectively dispatched economically[7].

The traditional single-layer wall thermal inertia model is quite different from the actual heat transfer process. This paper proposes a layered thermal inertia model to simulate the heat in the system in detail. In addition, this paper introduces the water-heating network model considering the piecewise fiction coefficient.

For the above problems, this paper first introduces multi-layer wall thermal inertia model as a thermal model of the building integrated energy system. Then this paper introduces the water-heating network model considering the piecewise fiction coefficient. Based on the building thermal inertia model combined with grid constraints and water-heating network constraints, an optimized plan for the RIES is established. Finally, the analysis results based on the IEEE-13 bus system show that the multi-layer wall thermal inertia model proposed in this paper can accurately describe the heat transfer process. The proposed RIES optimization scheme can reduce the users' electricity purchase cost while maintaining indoor comfort. It can also optimize the power flow of distribution network, which is of great significance to the safe and stable operation of power system.

\section{Thermal Model of RIES}

In this paper, wall and floor are modeled according to the thermal inertia model. We take the wall as an example to introduce the thermal inertia model.

\subsection{Multi-layer Thermal inertia model}

Reference[8] proposed to use the thermal resistance and thermal capacity model to simulate the heat transfer process of the room. The traditional thermal inertia model does not consider the heat transfer process inside the wall, so there is a big gap with the actual process. In order to simulate the heat absorption and release process of the

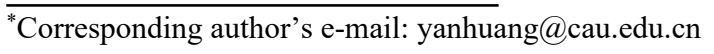


wall in the actual buildings energy system in more detail, this paper subdivides the interior of the wall into the outside, middle and inside to construct a new thermal inertia model, as shown in figure 1, the arrow indicate the heat transfer process.

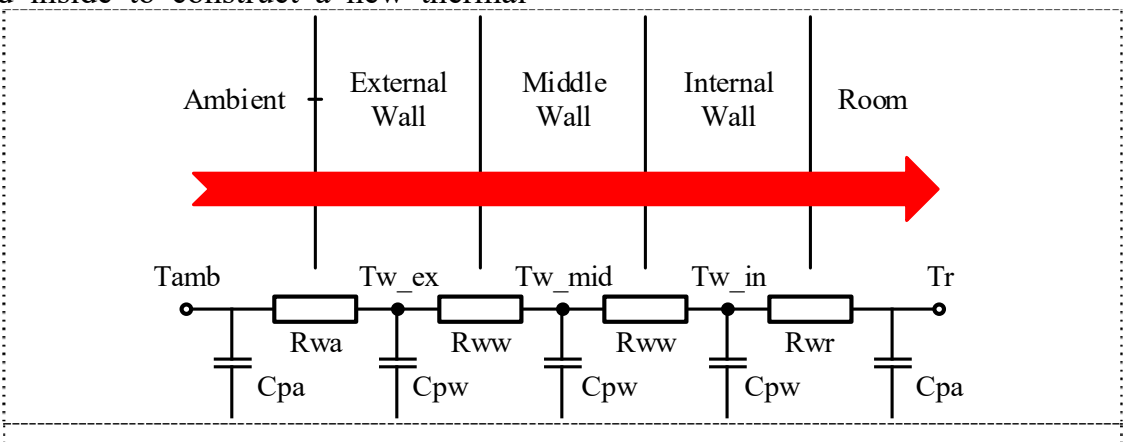

Figure 1. Multi-layer wall thermal inertia model.

Suppose that as the heat passes through the wall, each wall will absorb some of the heat and then release it. Therefore, in the thermal inertia model of multilayer wall,

$$
\begin{aligned}
& \frac{d T_{r}(t)}{d t}=\frac{1}{M_{a} \times C p_{a}} \times\left(\frac{d Q_{a b_{\_} a}(t)}{d t}-\frac{d Q_{w_{-} i n_{-} r}(t)}{d t}-\frac{d Q_{a c}(t)}{d t}\right) \\
& \frac{d T_{w_{-} e x}(t)}{d t}=\frac{1}{M_{w} \times C p_{w}} \times\left(\frac{d Q_{\text {gain_w_ex }}(t)}{d t}+\frac{d Q_{g_{\text {gain_w }} \text { mid }}(t)}{d t}\right) \\
& \frac{d T_{w_{-} \text {mid }}(t)}{d t}=\frac{1}{M_{w} \times C p_{w}} \times\left(\frac{d Q_{\text {gain_w_mid }}(t)}{d t}+\frac{d Q_{\text {gain_w_in }}(t)}{d t}\right) \\
& \frac{d T_{w_{-} i n}(t)}{d t}=\frac{1}{M_{w} \times C p_{w}} \times\left(\frac{d Q_{\text {gain_w_in }}(t)}{d t}+\frac{d Q_{w_{-} i n_{-} r}(t)}{d t}\right)
\end{aligned}
$$

where $T_{r}, T_{a m b}$ are the room temperature and ambient temperature respectively; $T_{w_{e x}}$ is external wall temperature; $T_{w_{-} \text {mid }}$ is middle wall temperature; $T_{w_{-} i n}$ is internal wall temperature. $C p_{a}$ is the equivalent heat capacity of air; $C p_{w}$ is the equivalent heat capacity of wall; $M_{a}$ is the mass of air; $M_{w}$ is the mass of wall; $Q_{\text {gain_w_ex }}(t), Q_{\text {gain_w_mid }}(t), Q_{\text {gain_w } w_{-} i n}(t)$, $Q_{w_{-} i n_{-} r}(t)$ is the heat of the outer wall, middle wall, inner wall and indoor air obtained from the adjacent medium; $Q_{a m b \_a}(t)$ is the heat from ambient to room; $Q_{a c}(t)$ is the heat generated by the air-conditioning.

In this paper, the energy efficiency coefficient is multiplied by the electric power of the air-conditioning to calculate the cooling capacity generated by the airconditioning [9].

\subsection{Water-heating network model}

For water-heating network model, we assume that it has only one heat source and one loop.

We define three types of temperatures variables [10]: The supply temperature $T_{s}$ is the temperature of hot water flowing into the load node. The outlet temperature $T_{o}$ is the temperature of hot water flowing out of the node. The return temperature $T_{r}$ is the temperature of hot water following the mixing of hot water flowing out of the load node with hot water in other pipelines.

The thermal power is:

$$
\Phi_{n}=C_{p} m_{q}\left(T_{s}-T_{o}\right)
$$

where $\Phi_{n}$ is the thermal load power at load node and $C_{p}$ is the specific heat of water.

As hot water flows from the beginning to the ending of the pipeline, the temperature of the hot water drops. Supposing that the thermal model does not have the thermal dynamic process. The relationship between the start and end temperatures is given by [11]:

$$
T_{\text {end }}=e^{-\frac{\lambda L}{m C_{p}}}\left(T_{\text {start }}-T_{a}\right)+T_{\text {amb }}
$$

where $T_{\text {start }}$ and $T_{\text {end }}$ denote the node start temperature and node end temperature; $\lambda$ is the pipe line heat transfers coefficient. In order to clearly explain the formulation, we introduce three new variables: $T_{\text {start }}^{\prime}=T_{\text {start }}-T_{a m b}$, $T_{\text {end }}^{\prime}=T_{\text {end }}-T_{\text {amb }}$, and $\varphi=e^{-\frac{\lambda L}{m C_{p}}}$.

Equation (6) can be rewritten as follows:

$$
T_{\text {end }}^{\prime}=T_{\text {start }}^{\prime} \varphi
$$

The mass flow rate of the load node in water network should satisfy the flow continuity equation:

$$
\left(\sum m\right)_{\text {in }}-\left(\sum m\right)_{\text {out }}=m_{q}
$$


where $m$ denotes the pipeline mass flow rate vector, $m_{q}$ denotes the mass flow rate vector consumed by load node. As water flow encounters external resistance, head loss is inevitable because liquid is viscous. The relationship between the head loss $h_{f}$ and $m$ is:

$$
h_{f}=K|m| m
$$

where $K$ denotes pipeline drag coefficient. $K$ can be calculated using the friction coefficient $f$ as follows:

$$
K=\frac{8 L f}{D^{2} \rho^{2} \pi^{2} g}
$$

where $L$ denotes the pipeline length, $D$ denotes pipeline diameter, $\rho$ denotes the density of water, $g$ is the acceleration caused by gravity, and $f$ depends on the Reynolds number[12].

\subsection{Objective function}

The optimization goal is to minimize the daily building electricity bills under peak and valley pricing. The formulation is as follows:

$$
c=\sum_{t=1}^{T} c(n) P_{\text {building }}(n) \Delta t
$$

where $c$ indicates the daily building electricity bills; $c(n)$ is the electricity price at time $n ; P_{\text {building }}(n)$ is total electrical power of the room at time $n ; \Delta t$ is the time interval.

\section{Example analysis}

In this section, the wall thermal inertia model and waterheating network model are tested on modified IEEE-13 case, as shown in figure 6. In alternating current (AC) systems, the power flow model for on-load transformers,

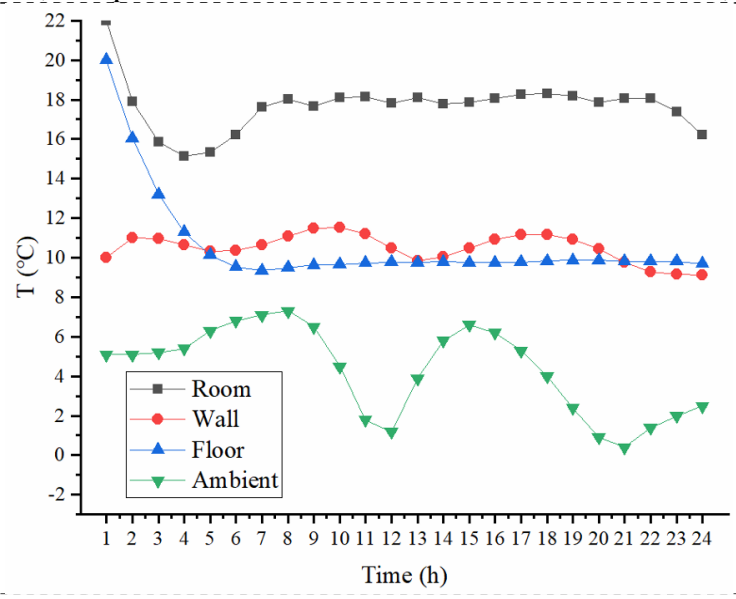

Figure 2. Ambient, wall, floor and room temperature curves in scheme 1. transmission lines, and distributed generator can refer to reference [13]. System structure and detailed parameters of test case can refer to [14]. There are 1000 smart houses connected to load bus 13, and each house uses the optimization strategy proposed in this paper. For waterheating network connected to bus 5 in this case, it has only one heat source and one loop.

Based on different thermal inertia models of the wall and floor, we compared two schemes, scheme 1 selects the single-layer wall and floor and scheme 2 selects the multilayer wall and floor. With a cycle of 24 hours a day, the time step is 1 hour.

The problem is mixed integer nonlinear programming (MINLP) problem. We use GAMS/KNITRO [15] to solve the MINLP problem, the version is 24.7.4.

\subsection{Scheme 1}

In scheme 1, the thermal resistance of wall (including windows) and floor is equivalent to total thermal resistance, respectively. Heat transfer within the walls is ignored. The initial temperature of the ambient temperature, wall, floor and room temperature is $5{ }^{\circ} \mathrm{C}$, $10^{\circ} \mathrm{C}, 20^{\circ} \mathrm{C}$ and $22^{\circ} \mathrm{C}$ respectively. The change curves of ambient, wall, floor and room temperature in scheme 1 are shown in figure 2.

\subsection{Scheme 2}

In scheme 2, the thermal resistance of wall (including windows) and floor is also equivalent to total thermal resistance, respectively. Heat transfer within the walls is ignored. The initial temperature of the ambient temperature, external wall, internal floor and room temperature is $5{ }^{\circ} \mathrm{C}, 5{ }^{\circ} \mathrm{C}, 20{ }^{\circ} \mathrm{C}$ and $22{ }^{\circ} \mathrm{C}$ respectively. The change curves of room, wall, floor and ambient temperature in scheme 2 are shown in figure 3.

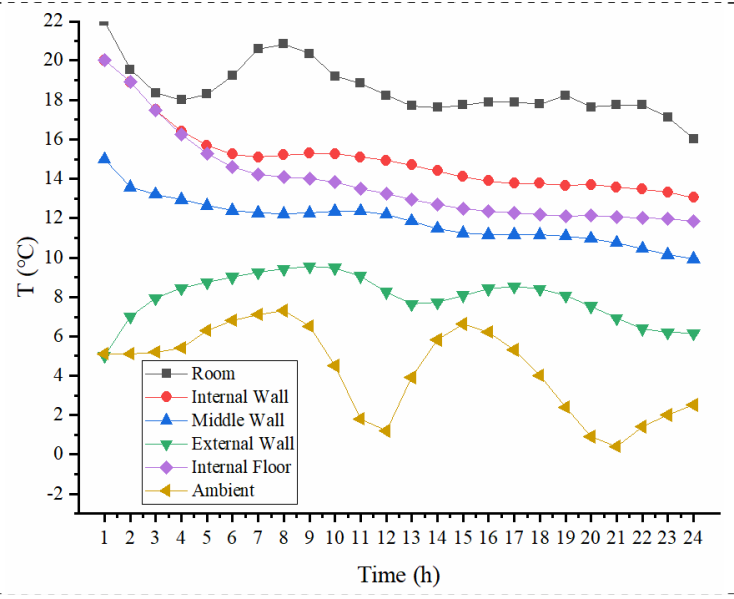

Figure 3. Room, wall, floor and ambient temperature curves in scheme 2. 


\subsection{Comparison of two schemes}

In this section, we compare the room temperature with 24 hours between the two schemes. As can be seen from

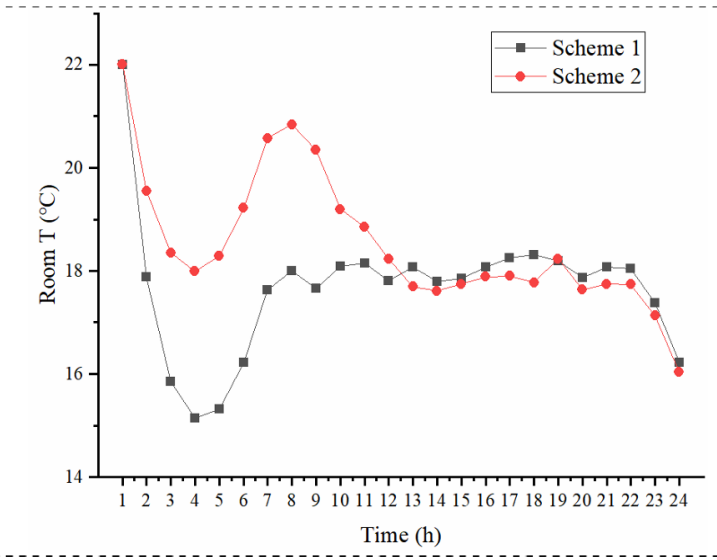

Figure 4. Room temperature change curves in scheme 1 and scheme 2 . figure 4, the room temperature within the time period of 1:00 10:00 of scheme 2 is higher than that of scheme 1. It shows that the multi-layer wall and floor thermal inertia model has better heat storage performance than singlelayer model at the same outdoor temperature.

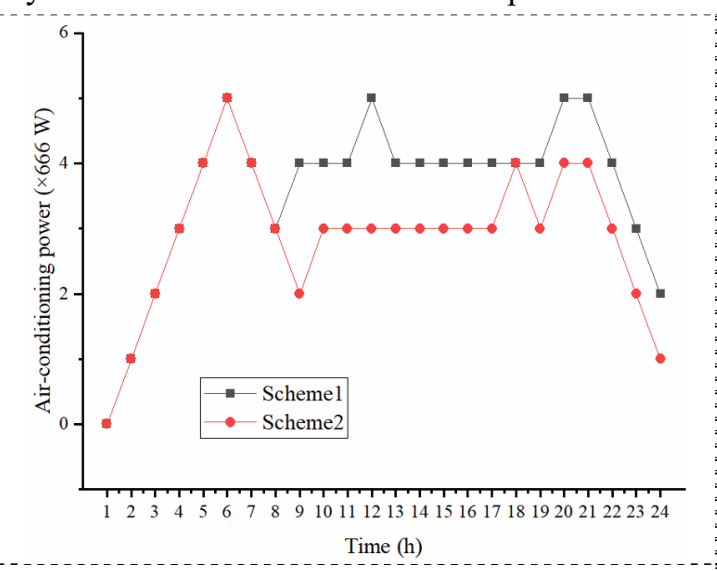

Figure 5. Air-conditioning power change curves in scheme 1 and scheme 2 .
We compare the air-conditioning power change curves in the two schemes within 24 hours. As shown in figure 5, we can see that due to the poor heat storage performance

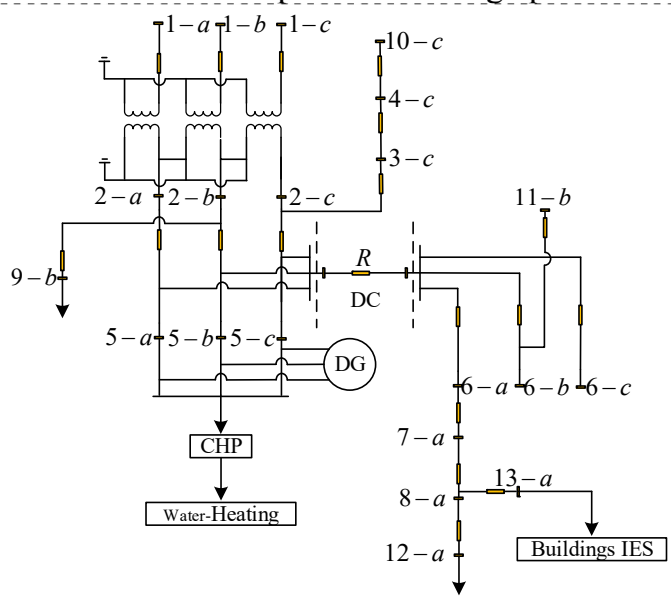

Figure 6. Diagram of modified IEEE-13 bus RIES structure. of the single-layer model in scheme 1, the indoor heat loss is rapid, which leads to the increase of air conditioning power consumption.

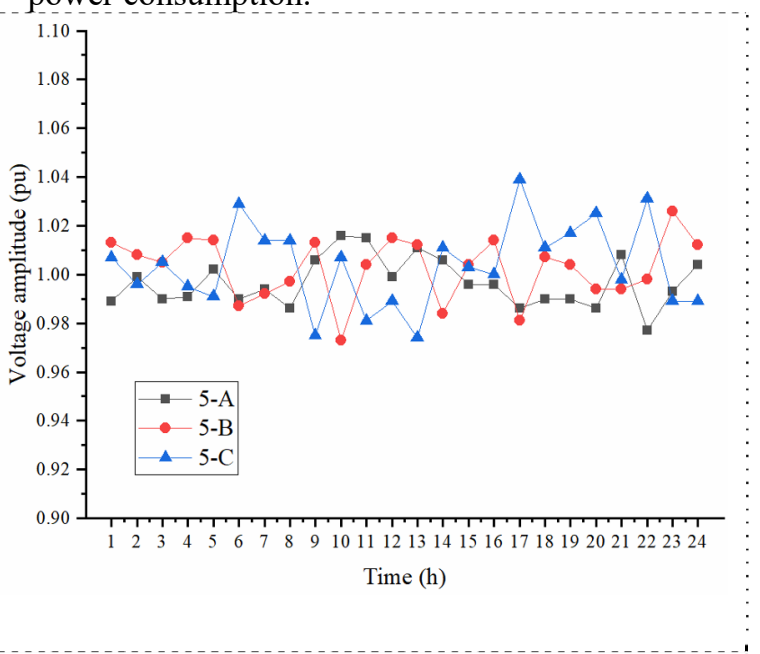

Figure 7. Bus 5 voltage amplitude of scheme 2.
Figure 7 is the calculation result of the bus 5 voltage amplitude within 24 hours of the day when the RIES optimization scheme 2 is adopted in the modified IEEE13 test case. It can be seen that the power fluctuations at each node in the network cause the three-phase voltage amplitude of the node to fluctuate within a certain range, but the voltage amplitudes of bus 5 at all times are within the range allowed by the safe operation of the system, and can be maintained. This verifies the feasibility of the RIES optimization scheme proposed in this paper.

\section{Conclusion}

This paper has developed a multi-layer wall thermal model based on thermal inertia model and water-heating network model considering the piecewise fiction coefficient. Based on the buildings thermal inertia model combined with grid constraints and water-heating network constraints, this paper establishes an optimal plan for the RIES. The multi-layer wall thermal model has better performance, which is reflected in more consistent with the actual temperature changes. And the proposed RIES optimization scheme based on multi-layer wall thermal model can reduce the electricity purchase cost while 
maintaining indoor comfort. The robustness and effectiveness of the RIES optimization scheme proposed in this paper have been verified by the test cases.

\section{References}

1. Wang, Y., Wang, Y., Huang, Y., Yu, H., Du, R., Zhang, F., Zhang, F., Zhu, J. (2019). Optimal Scheduling of the Regional Integrated Energy System Considering Economy and Environment. IEEE Transactions on Sustainable Energy, 10(4): 19391949.

2. Sharma, S., Verma, A., Xu, Y., Panigrahi, B. K. (2021). Robustly Coordinated Bi-Level Energy Management of a Multi-Energy Building Under Multiple Uncertainties. IEEE Transactions on Sustainable Energy, 12(1): 3-13.

3. Chen, H., Meng, K., Qiu, J., Dong, Z. Y. (2018). Expansion Co-Planning of Integrated ElectricityHeat-Gas Networks in District Energy Systems. In: 2018 IEEE Power \& Energy Society General Meeting (PESGM). Portland. pp. 1-5.

4. Saelens, D., Parys, W., Baetens, R. (2011). Energy and comfort performance of thermally activated building systems including occupant behavior. Building and Environment, 46(4): 835-848.

5. Wang, X. W., Gong, Y. F. (2017). User-side load fast precise dispatching model based on contracts and direct load control. In: 2017 2nd International Conference on Power and Renewable Energy (ICPRE). Chengdu. pp. 747-751.

6. Li, Z., Wu, W., Zhang, B., Tai, X. (2018). Capacity guaranteed control method for air conditioning cluster joining power grid frequency regulation. The Journal of Engineering, 2018(17): 1884-1888.

7. Zhu, L., Wu, W., Li, Z., Liu, W., Li, L. (2019). AirConditioning Optimal Scheduling Based on Finite Difference Thermal Model. In: 2019 IEEE Sustainable Power and Energy Conference (ISPEC). Beijing. pp. 469-476.

8. Haiming W., Ke M., Feng J., Zhao Y., Verbič, G., Zhao X, Wong, K. (2013). Demand response through smart home energy management using thermal inertia. In: 2013 Australasian Universities Power Engineering Conference (AUPEC). Hobart. pp. 1-6.

9. Hundy, G., Trott, A., Welch, T. (2008) . Refrigeration and Air-Conditioning.

10. Steer, K., Wirth, A., Halgamuge, S. (2011). Control period selection for improved operating performance in district heating networks. Energy and Buildings, 43(2-3): 605-613.

11. Yang, B., Zhou, X., Cao, X., Zhi, Y., Ding, Y. (2018). A power flow analysis method for the integrated electricity-heat system in distribution network based on forward/backward iterations. In: 2018 2nd IEEE Conference on Energy Internet and Energy System Integration (EI2). Beijing. pp. 1-5.
12. Liu, X., Wu, J., Jenkins, N., Bagdanavicius, A. (2016). Combined analysis of electricity and heat networks. Applied Energy, 16(2): 1238-1250.

13. Ju, Y., Wang, J., Zhang, Z., Huang, Y. (2020). A Calculation Method for Three-Phase Power Flow in Micro-Grid Based on Smooth Function. IEEE Transactions on Power Systems, 35(6): 4896-4903.

14. Kersting, W. H. (2001). Radial distribution test feeders. 2001 IEEE Power Engineering Society Winter Meeting. Conference Proceedings, 2(2): 908912.

15. Byrd, R., Nocedal, J., Waltz, R. A. (2006). Knitro: An Integrated Package for Nonlinear Optimization. Large-Scale Nonlinear Optimization 83(5): 35-59. 